\title{
Physical modeling of the formation of clathrate hydrates of methane
}

\author{
A. Drobyshev, A. Aldiyarov, V. Kurnosov, K. Katpaeva, E. Korshikov, \\ D. Sokolov, A. Shinbayeva, and A. Timchenko \\ al-Farabi Kazakh National University, Almaty 050038, Kazakhstan \\ E-mail: Andrei.Drobyshev@kaznu.kz
}

Received January 8, 2015, published online April 23, 2015

\begin{abstract}
Nowadays natural gas hydrates attract special attention as a possible source of fossil fuel. According to various estimates, the reserves of hydrocarbons in hydrates exceed considerably explored reserves of natural gas. Due to the clathrate structure the unit volume of the gas hydrate can contain up to 160-180 volumes of pure gas. In recent years interest to a problem of gas hydrates has considerably increased. Such changes are connected with the progress in searches of the alternative sources of hydrocarbonic raw materials in countries that do not possess the resources of energy carriers. Thus gas hydrates are nonconventional sources of the hydrocarbonic raw materials which can be developed in the near future. At the same time, mechanisms of methane clathrate hydrates formations have not reached an advanced level, their thermophysical and mechanical properties have not been investigated profoundly. Thereby our experimental modeling of the processes of formation of methane clathrate hydrates in water cryomatrix prepared by co-condensation from the gas phase onto a cooled substrate was carried out over the range of condensation temperatures $12-60 \mathrm{~K}$ and pressures $10^{-4}-10^{-6}$ Torr. In our experiments the concentration of methane in water varied in the range of 5-90\%. The thickness deposited films was 30-60 $\mu \mathrm{m}$. The vibrational spectra of two-component thin films of $\mathrm{CH}_{4}+\mathrm{H}_{2} \mathrm{O}$ condensates were measured and analyzed.
\end{abstract}

PACS: 61.50.-f Crystal structure;

78.30-j Infrared and Raman spectra;

68.35.Rn Phase transitions and critical phenomena.

Keywords: methane clathrate, cryocondensation, IR-spectroscopy, cryomatrix.

\section{Introduction}

Interest to study methane hydrates is caused by their application importance and intriguing basic properties. When we speak about practical application of the knowledge, which is connected with the formation processes and methane clathrates properties, first of all we must remember that the bulk of methane deposits are concentrated at substantial depths of the World Ocean and exist in the form of clathrates. Extraction of these minerals is an important scientific and applied task.

Moreover, there are a plenty of questions, which are connected with the interaction of methane molecules with other substances [1]. In particular, it is the question how methane molecules interact with water molecules, when the quantum features of methane, such as the mixing of oscillatory and rotational modes, are of primary importance. The second circumstance is connected with the ability of water molecules to form networks of hydrogen bonds. The combination of these properties results in the formation of methane clathrates, i.e., systems, which consist of methane molecules, surrounded by water cages, which are linked by hydrogen bonds.

This study of clathrate systems has the following peculiarities. We suppose that the behavior of methane in the water environment at low pressures (as if carried out at a depth of 2-3 km) can be reasonably modeled by the method of cryomatrix isolation with high concentrations of methane. We suppose that the position of a methane molecule in the lattice of water ice resembles that in a clathrate. Ice can create the same static influence, as water at a significant depth. In this case we have the object we need to study and can carry out our research in vitro.

Analysis of vibrational ranges of two-component mixes (water and methane), which were formed at various conditions, serve as the basis of our research technique. The fea- 
tures to be found can be interpreted as manifestations of methane bounded states in solid solutions with water. Thus, the co-condensation method gives opportunities to vary experimental conditions over a wide range and also to study possible thermostimulated processes and effects.

\section{Experimental}

Analysis of the absorptive amplitude of the band, which corresponds to the vibrations of the methane molecule in the unbounded state, is at the core of the procedure for obtaining information about the state of methane molecules in the matrix of different gases. The measurements were carried using the setup, depicted in Fig. 1. The main unit of the system is a cylindrical vacuum chamber (1) with the diameter and height of $450 \mathrm{~mm}$. Pumping out of the vacuum chamber was carried out by turbo-molecular pump Turbo-V-301 (2), which was connected to the chamber through a sliding vane gate valve CFF-100 (3). A dry spiral pump SH-110 (not shown) was used as a fore vacuum pump. The ultimate vacuum in the chamber reached the value of $10^{-8}$ Torr. Pressure measurements was carried out using a wide-range pressure transducer FRG-700 (4) with an AGC-100 controller.

The Gifford-McMahon microcryogenic system (5) was located in the center of the chamber. A mirror substrate (6), which serves as the condensation surface for methane and water mixtures was mounted on the top flange of the microcryogenic system. The substrate was made of copper, the working surface of which was covered with silver. The diameter of the substrate is $60 \mathrm{~mm}$. The minimum temperature of condensation is $12 \mathrm{~K}$. The temperature measurements were carried out by silicon sensor TS 670-1.4

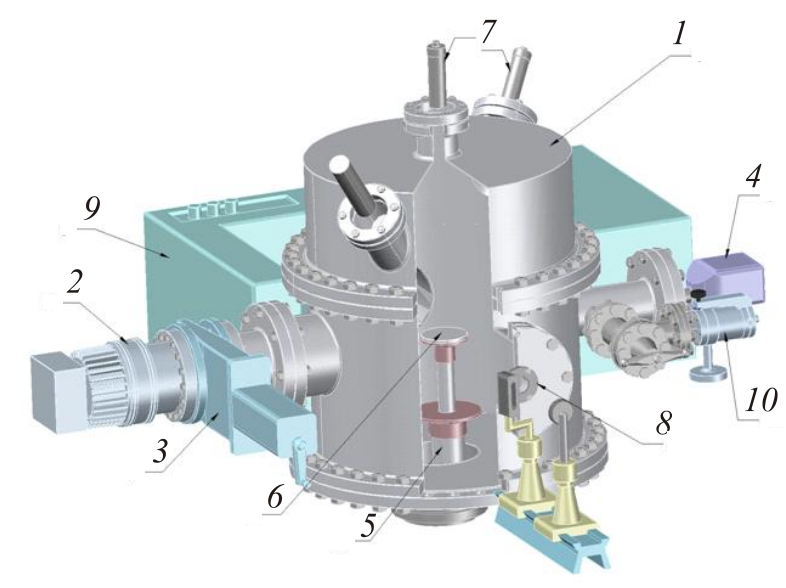

Fig. 1. Experimental installation: vacuum chamber (1); pump Turbo-V-301 (2), sliding vane gate valve CFF-100 (3); pressure transducer FRG-700 (4); microcryogenic system GiffordMcMahon (5); substrate (6); photomultiplier (7); optical channel (8); IR-spectrometer (9); leakage system (10). using a temperature controller M335/20c. Measurements of thicknesses and rates of condensation were carried out using a double-beam laser interferometer which was based on a photo-electron-multiplier P25a-SS-0-100 (7). IR absorption spectrum was measured in the frequency range $400 \mathrm{~cm}^{-1}-4200 \mathrm{~cm}^{-1}$.

A calibrated volume (not shown) was used to obtain a mixture of the test substance with a matrix gas. First the gas (methane) was let into the volume to a pressure below the equilibrium pressure at a given ambient temperature. Typically, the pressure was 1 to 1.5 Torr. Thereafter, the calibration volume was filled with water vapor until the required work pressure was reached. Pressure controller PR 4000 (MKS) was employed during the preparation of the mixtures with an accuracy of pressure measurements of 0.01 Torr.

The experimental procedure was as follows. The vacuum chamber was pumped down to a pressure of $10^{-8}$ Torr, then the substrate was protected by a plate to prevent contamination and cooled up to $12 \mathrm{~K}$. The operating pressure of the mixture in the chamber $10^{-5}$ Torr was set using the leakage system (10), the substrate was bared and the process of film cryocondensation begun. The process was monitored using a double-beam laser interferometer. Once the sample thickness reached of about 2-3 $\mu \mathrm{m}$ the gas filling process was stopped, the pressure in the chamber being approximately $10^{-8}$ Torr. Next, the vibrational spectrum of the sample was measured, where upon the IR-spectrometer was set to the needed frequency and the interferometer signal was measured during 30-40 min at a constant temperature, which was equal to the condensation temperature of $16 \mathrm{~K}$. Thus, the state of the sample was analyzed over time at a constant temperature.

Further measurements were carried out using two methods. In one case, the stepwise heating process of the sample was carried out $0.5-1 \mathrm{~K}$, the reflectance spectra being measured a fixed temperature. In the second case, we applied a continuous heating of the sample, the rate of which was determined by the natural heat inflow to the substrate with the microcryogenic machine switched off. In this case, the IR-spectrometer signal was measured at a fixed frequency in the vicinity of the characteristic vibration frequencies of the water molecule. Changes in the signal are reflected transformations in the test sample.

\section{Results and discussion}

In the present study we used methane gas produced by company IHSAN TECHNOGAZ. The gas purity was $99.99 \%$. Figure 2 shows the vibrational absorption spectrum of methane in the gas (bottom spectrum) and solid (upper spectrum) states. The former spectrum was measured at 4 Torr and $300 \mathrm{~K}$. The solid sample was obtained by condensation at $10^{-5}$ Torr and a substrate temperature of $16 \mathrm{~K}$. The thickness of the sample was $5 \mu \mathrm{m}$. 


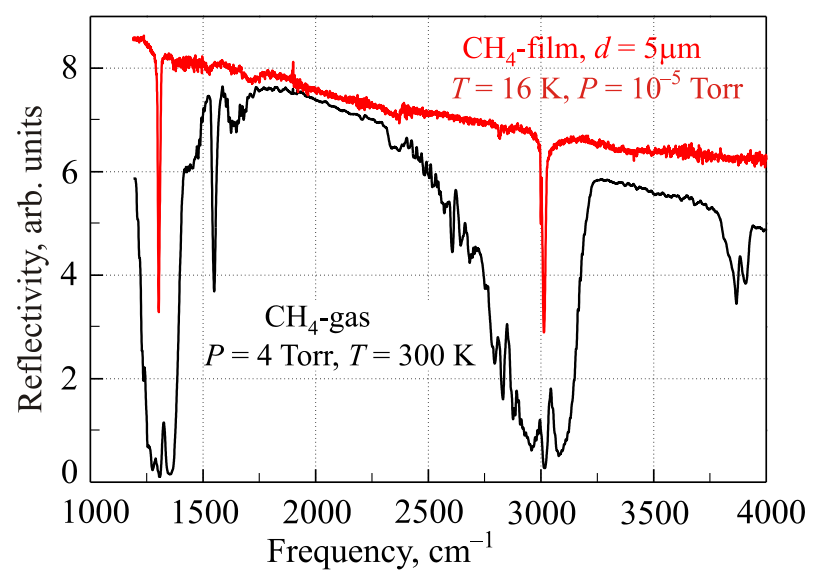

Fig. 2. (Color online) The rotational-vibrational spectrum of methane in gas and solid states.

We will not go into much detail of those spectrums, bearing in mind that the character of these measurements is predominantly calibration. We only note that methane condensation entails freezing of the rotational subsystem and the spectrum consists of two fairly narrow absorption bands. The maximum of the band for $\mathrm{C}-\mathrm{H}$ stretch vibration line is at $3016 \mathrm{~cm}^{-1}$. The minimum of the band that corresponds to deformation vibrations is at $1306 \mathrm{~cm}^{-1}$. The information of these spectra is necessary for analyzing the state of methane molecules in the solid solution with water.

Methane hydrates formation process implies presence of certain features of methane molecules and water ice lattice interactions. It is appropriate to investigate the effect of neutral cryomatrix on the methane vibration spectrum to correctly assess this effect. In addition, these measurements are not the main purposes of our studies, and their character is calibrating.

Figure 3 shows the results for a 5\% methane solution with nitrogen and argon: the data for the range of $\mathrm{C}-\mathrm{H}$ stretching vibrations (a); the data for deformation vibrations (b). These data are compared with the corresponding absorption bands of pure methane cryocondensate. As seen in Fig. 3, the stretching and deformation bands of methane molecules in solution with argon and nitrogen are noticeably shifted to higher frequencies compared to pure methane, which reflects the effect of the matrix lattice. Interestingly, the valence band shift of about $9 \mathrm{~cm}^{-1}$ is almost the same for both. At the same time, the deformation bands (cf. Fig. 3(b)) are shifted by $6 \mathrm{~cm}^{-1}$ and $9 \mathrm{~cm}^{-1}$ methane in the argon and methane matrices, respectively.

In this article we also present results for methane-water cryocondenced films for methane concentrations from 5 to $90 \%$. The experiments included the following stages. Mixtures of methane gas and water vapor of a certain concentration at a total pressure of $10^{-5}$ Torr was admitted into the camera (preliminarily evacuated to $10^{-8}$ Torr) to be condensed. The resulting sample thickness was $10-15 \mu \mathrm{m}$. It should be noted that changes in mixture composition
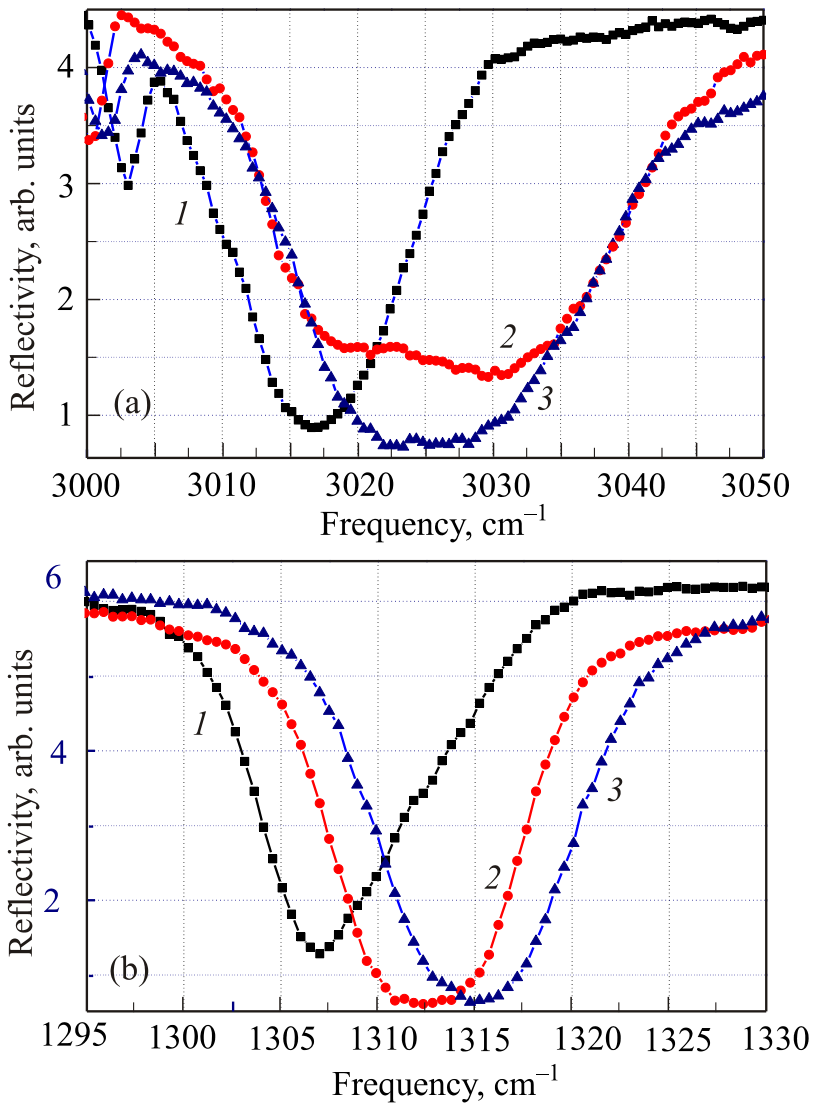

Fig. 3. (Color online) IR spectra of pure methane and its 5\% mixtures with nitrogen and argon in the ranges of $\mathrm{C}-\mathrm{H}$ stretching (a) and deformation (b) vibrational frequencies. $\mathrm{CH}_{4}, T_{c}=16 \mathrm{~K}(1)$; $5 \% \mathrm{CH}_{4}+\mathrm{Ar}, T_{c}=16 \mathrm{~K}(2) ; 5 \% \mathrm{CH}_{4}+\mathrm{N}_{2}, T_{c}=16 \mathrm{~K}(3)$

entailed changes in film thickness provided that the methane mass content remained approximately the same. Absorption spectra were measured after the necessary film parameters have been reached. The two measurement procedures were the same as employed previously [2,3]. In the first case, the spectra were recorded at a fixed substrate temperature as the film grew thicker. According to the second technique the frequency of our IR-spectrometer was set to its maximum value for the methane absorption band. As a rule, we set the frequency of deformation vibrations because, unlike the valence $\mathrm{C}-\mathrm{H}$ vibrations, the absorption band at this frequency is separated from the absorption bands of water. Further we measured the absorption amplitude at the chosen frequency in the course of a slow warmup until the sample evaporated. Such thermograms provide continuously information about the state of the samples.

The pressure in the vacuum camera is another parameter, which was measured in our experiments. To vary the pressure, the pumping valve of the camera was slightly opened to pump out the residual gases which are the consequences of inleakage. Thus, a spontaneous increase in pressure in the camera may be connected with the thermodesorption processes occurring in the sample, which are reflected in the characters of the thermograms obtained. 
This method of thermal desorption was widely used earlier by us and some other authors [4-6]. Physical modeling of methane hydrate formation implies the measurement of oscillatory ranges of the condensed films and the subsequent analysis of their transformations under warm-up.

The vibrational range of the sample, which consisted of $25 \%$ of methane and $75 \%$ of water is presented in Fig. 4. The absorption band at $1320 \mathrm{~cm}^{-1}$ corresponds to the deformation (or rotational ) vibrations of $\mathrm{C}-\mathrm{H}$ bonds of methane. The wide peak at $1676 \mathrm{~cm}^{-1}$ is related with deformation vibrations of the water molecule. The narrow peak at $3020 \mathrm{~cm}^{-1}$ corresponds to stretching vibrations of $\mathrm{CH}_{4}$, the next wide peak, centered at $3300 \mathrm{~cm}^{-1}$, corresponds to the $\mathrm{OH}$ bond of the water molecule. The nature of the peak at $3670 \mathrm{~cm}^{-1}$ is unusual. Traditionally, the vibrations in this frequency range are refered to as quasi-free vibrations of monomers or dimers of linear water molecules in cryomatrix [7-9]. In the cited works this peak was a narrow band with a pronounced minimum, whereas in the present studies the band at $3670 \mathrm{~cm}^{-1}$ and the deformation peak of water at $1676 \mathrm{~cm}^{-1}$ have a complex structure that may be due to the peculiarities of interactions between water and methane molecules. As seen in Fig. 4, the absorption bands of methane in the $25 \%$ mixture is slightly "blue" shifted relative to pure solid methane, the value for bending vibrations of about $14 \mathrm{~cm}^{-1}$ and for $\mathrm{CH}$ stretching vibrations of approximately $5 \mathrm{~cm}^{-1}$. It is virtually identical to the data for other matrices (Fig. 2). Thus, we can conclude that the state of methane molecules, its vibrational spectrum dependent weakly on the composition of the mixtures discussed in this article.

Infrared spectra of the samples condensed at $T=16 \mathrm{~K}$ were measured; the samples were subsequently warmed with intervals of $2-5 \mathrm{~K}$ until complete evaporation of the sample at about $180 \mathrm{~K}$. It was found that up to $160 \mathrm{~K}$ the methane vibration band persists. Here we note that the

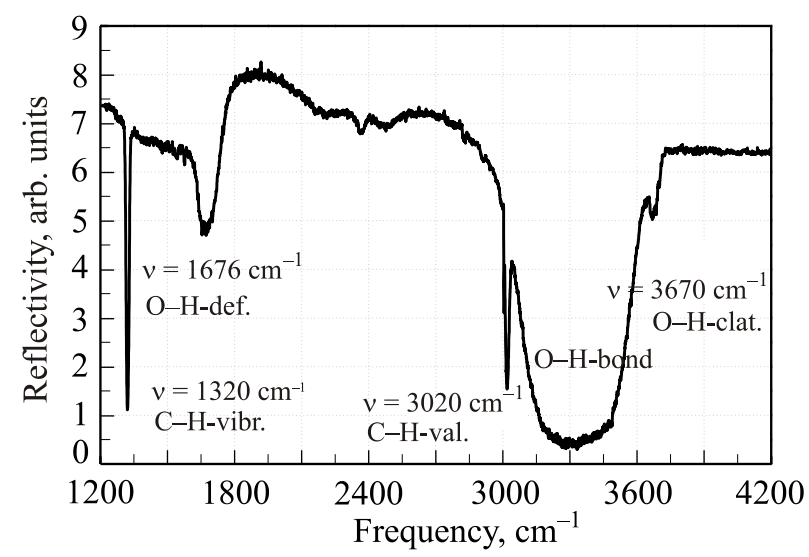

Fig. 4. IR-spectrum of the film $\mathrm{CH}_{4}(25 \%)+\mathrm{H}_{2} \mathrm{O}(75 \%)$ methane and $75 \%$ water cryovacuum condensate. The temperature of condensation $T=16 \mathrm{~K}$; the pressure $P=10^{-5}$ Torr, film thickness $d=10 \mu \mathrm{m}$. methane pressures within the range $10^{-5}-10^{-6}$ Torr correspond to the equilibrium temperatures 36 to $38 \mathrm{~K}$.

This means that pure solid methane must have completely evaporated above these temperatures. We can reliably assume that in this case at least two bound states of methane in water exist; namely, the methane, adsorbed by cryocondensate layers of water, and the methane in hydrate cages. These two states of methane may reveal themselves at elevated temperatures in the form of characteristic absorption bands in the IR spectra.

As understood from our previous studies [10], monitoring at a fixed frequency can give more detailed information about thermally stimulated processes in cryocondensates. As noted above, because the methane deformation vibration band stands off other bands, it is more convenient to observe its behavior. The results of these measurements are presented in Fig. 5. These thermograms of methane and water at different compositions were obtained at the IRspectrometer frequency of $1320 \mathrm{~cm}^{-1}$. The thin lines are the absorption bands in question, which clearly demonstrates the position of measured values on the thermograms relatively to absorption bands, i.e., the presence of methane in the sample.

When analyzing the thermograms in Fig. 5, we should note the following basic facts.

1. Methane in a solid solution with water is present in the sample up to water evaporation temperature, i.e., to 160-170 K.

2. The character of the thermograms significantly depends on the methane concentration. The low methane concentrations in the gas phase lead to slower and less sharp changes in the amplitude of deformation bands.

3. The characteristic temperatures of the region of the most radical changes of the thermograms are $40 \mathrm{~K}$ (the sublimation temperature of pure methane [11]) and 135-140 K (the temperature of the amorphous ice water transition to the state of supercooled liquid SCL $[11,12])$.

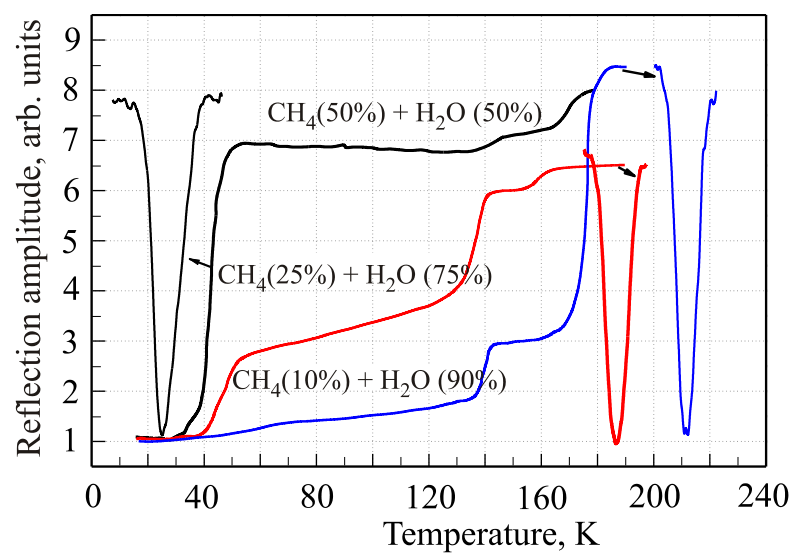

Fig. 5. (Color online) Thermally stimulated changes in the reflectivity at $v=1320 \mathrm{~cm}^{-1}$. Thermograms are presented for different methane-water compositions. The thin lines are the absorption bands of the appropriate methane deformation. 
Comparison of the thermograms with chamber pressure measurements at the same temperatures provides one more insight into the processes that take place in the samples with increasing temperature.

As can be seen from Figs. 5 and 6, the character of changes the amplitude of methane molecule deformation vibrations depends on the sample composition. However, it should be noted that the characteristic features of the thermograms for all methane concentrations are closely associated with the desorption peaks. Thus, the interpretation of the desorption peaks in combination with IR thermograms may play a crucial role in understanding of the processes in the samples. In particular, peak 1 in Figs. 6 and 7 (in Fig. 8 it misses), most likely, is not associated with changes in methane concentration in the sample, because its appearance at $20 \mathrm{~K}$ is not accompanied by changes in the amplitude of deformation vibrations. Apparently, this peak corresponds to desorption of the gas (possibly, nitrogen desorption), which recondenses from the film onto inoperative surfaces of the substrate. This fact was also mentioned in [13].

In Fig. 6, desorption peaks 1 and 2, as well as peak 2 in Fig. 7 and peak 1 in Fig. 8 are in full correspondence with the changes in the absorption amplitude of methane deformation vibrations at $1305 \mathrm{~cm}^{-1}$.

The beginning of pressure increase in the vicinity of $40 \mathrm{~K}$ coincides with the sharp reduction of absorption amplitude at the operating frequency. Further temperature increase in the interval from $40 \mathrm{~K}$ to approximately $58 \mathrm{~K}$ is accompanied by an increase of the desorbed gas pressure to a maximum value at $42-43 \mathrm{~K}$ with subsequent reduction to the original value $\left(5 \cdot 10^{-7}\right.$ Torr). Maximum values of desorption pressure for different samples were $2 \cdot 10^{-5}$ Torr in Fig. 6, 9·10 $10^{-6}$ Torr in Fig. 7, and $4 \cdot 10^{-6}$ Torr in Fig. 8. The higher was the methane concentration in the sample, the higher was the thermal desorption pressure step.

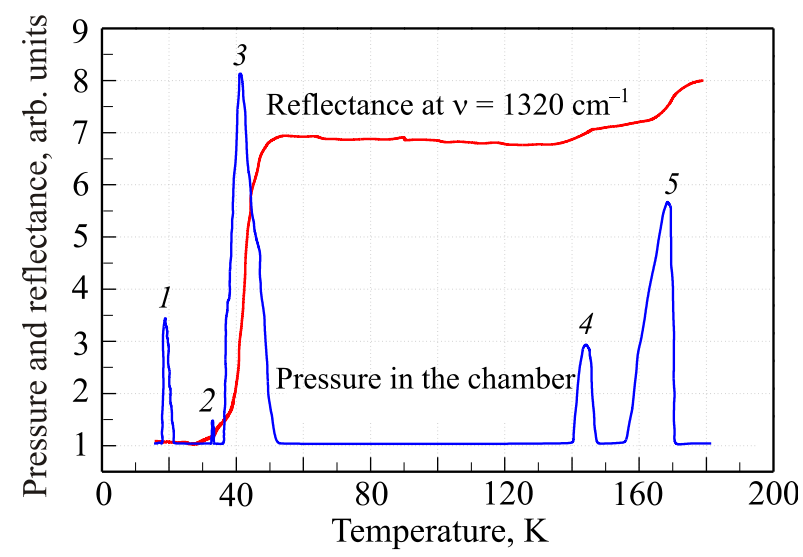

Fig. 6. (Color online) Changes in the reflectance of the $\left(50 \% \mathrm{CH}_{4}+\right.$ $+50 \% \mathrm{H}_{2} \mathrm{O}$ ) sample at the frequency of observation $1305 \mathrm{~cm}^{-1}$ (upper curve) and in the pressure in the chamber (bottom curve) during the heating process. Condensation temperature is $16 \mathrm{~K}$, the sample thickness is $10 \mu \mathrm{m}$.

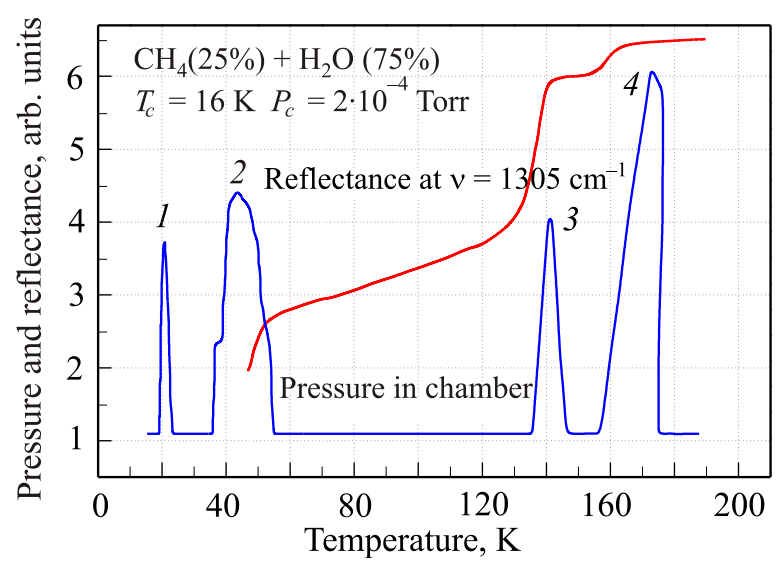

Fig. 7. (Color online) Changes in the reflectance of the $(25 \%$ $\mathrm{CH}_{4}+75 \% \mathrm{H}_{2} \mathrm{O}$ ) sample at $1305 \mathrm{~cm}^{-1}$ (upper curve) and in the pressure in the chamber (bottom curve) during the heating process. Condensation temperature $16 \mathrm{~K}$, the sample thickness $10 \mu \mathrm{m}$.

The obvious fine structure presence of the desorption peaks in different samples suggests that the processes that occur in this temperature range, are more complex than simple desorption of cryocaptured methane molecules. However, in this article we do not discuss these issues, because special studies of this phenomenon are needed for clarification.

Further warm-up to $60-130 \mathrm{~K}$ entails a flat spectrometer signal (see Fig. 6) or a monotonic decrease of the methane concentration, as shown in Fig. 7 and 8. Obviously, such a behavior is associated with changes in the composition of the samples. The appearance of a desorption peak and the respective sharp change in absorption amplitude in the temperature range 130-145 $\mathrm{K}$ were observed. The maximum desorption pressures for different samples were $2 \cdot 10^{-6}$ Torr in Fig. 6, 7·10 ${ }^{-6}$ Torr in Fig. 7, and $8 \cdot 10^{-6}$ Torr in Fig. 8. Thus, the desorption pressures are almost the

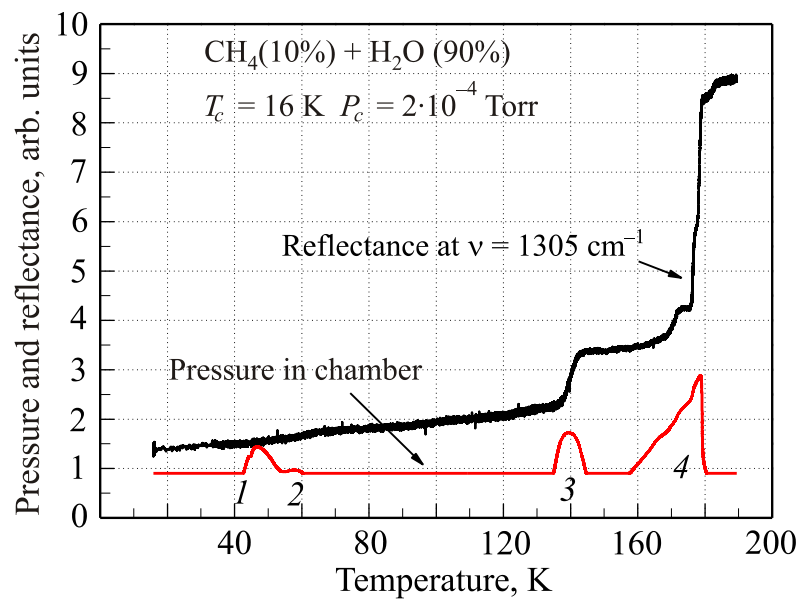

Fig. 8. (Color online) Changes in the reflectance of the $\left(10 \% \mathrm{CH}_{4}+\right.$ $+90 \% \mathrm{H}_{2} \mathrm{O}$ ) sample at $1305 \mathrm{~cm}^{-1}$ (upper curve) and in the pressure in the chamber (bottom curve) during the heating process. Condensation temperature $16 \mathrm{~K}$, the sample thickness $10 \mu \mathrm{m}$. 
same independent of methane concentrations from $25 \%$ to $10 \%$, while for the sample with a methane concentration of $50 \%$ this pressure is almost smaller by a factor of 3 .

The temperature range $145-160 \mathrm{~K}$ is characterized by stabilization processes occurring in all the three samples. The pressure in the chamber and the amplitude of methane deformation vibrations remain almost unchanged. Starting from $160 \mathrm{~K}$, a sharp increase in the chamber pressure and a simultaneous decrease in the absorption band amplitude were documented. This process proceeds until complete evaporation of the samples at $180 \mathrm{~K}$. It should be noted that methane remains present in the samples within the entire temperature range from $160 \mathrm{~K}$ to $180 \mathrm{~K}$.

\section{Conclusions}

Our studies have shown that a two-component solid film was formed in the process of methane and water cocondensation on the substrate at the temperature of $T=$ $=16 \mathrm{~K}$. It is practically identical to the data for nitrogen and argon matrices, from which we can conclude that the state of methane molecule and its vibrational spectrum are weakly dependent on the composition in the discussed mixtures.

At this stage of research we can make some assumptions relatively the status of methane molecules in the "matrix" of water which are based on the comparison of the thermal desorption curves and thermograms of the absorption amplitudes changes of methane characteristic vibration frequencies. In our view, it is natural to assume that under these conditions cryoprecipitated methane in solid solution with water can exist in three states. Firstly, it is a condensed state, i.e. solid phase of methane. Secondly, the methane can be in an adsorbed state. The role of absorbent is played by the amorphous solid water (ASW). This state is typical for water cryovacuum condensates formed at $T=16 \mathrm{~K}$ [14]. Thirdly, methane may be in a bound state with water molecules, which form clathrates. This, indeed, is the subject of our study. In this paper we attempt to determine the temperature ranges of these states, based on the properties of amorphous solid water ASW and comparing obtained thermograms of desorption and absorption amplitudes of methane deformation vibrations. As it can be seen in Figs. 6-8 the data for more detailed analysis can be divided into four main temperature ranges.

Temperature range 30-58 $\mathrm{K}$. In our opinion, the cause of desorption peak and a sharp change of the absorption amplitude at observation frequency of $v=1305 \mathrm{~cm}^{-1}$ is the evaporation of solid methane, which was condensed at $T=16 \mathrm{~K}$. This assumption is consistent with the values of methane equilibrium parameters (pressure, temperature) on the phase diagram.

Temperature range $60-130 \mathrm{~K}$. There is a monotonic decrease in the concentration of adsorbed methane in the sample, which is associated with a decrease in the adsorp- tion capacity of the amorphous film of water at higher temperatures. The process is extended in time, so that the desorbed methane pumped from the chamber without desorption peak formation.

Temperature range 130-145 K. Observed sharp changes of the measured parameters are related, in our opinion, to the restructuring of the solid phase from the water in ASW through the intermediate state SCL to cubic ice [4,14]. Due to the presence of an intermediate state SCL adsorbed methane receives necessary diffusion mobility to move to the phase boundary and subsequent desorption.

Temperatures from $145 \mathrm{~K}$ to sample evaporation point. In the range 145-160 $\mathrm{K}$ the methane concentration in the sample stays almost unchanged. Thus, the quantity of methane in the samples is sufficiently large. In particular, if we judge by the value of the absorption amplitude, there is at least $60 \%$ of original methane concentration in $10 \%$ sample at $160 \mathrm{~K}$ (see Fig. 5). At these high temperatures, methane can exist in the film only in the bound state with water, namely in the form of clathrates. Increase of the temperature above $160 \mathrm{~K}$ leads to a decrease of methane concentration, which is most likely connected with the transition of cubic ice $I_{c}$ to the regular hexagonal state $I_{h}$, which is apparently accompanied by a partial destruction of the clathrates. Narrow temperature range 172-176 K (Fig. 8) with constant values of the methane concentration corresponds to the existence of hexagonal ice containing methane clathrates. Further increase of the temperature leads to an evaporation of water film with simultaneous change of methane concentration, i.e., its evaporation. The fact that methane leaves the sample together with the evaporating water confirms methane clathrates hydrates presence in the samples.

Thus, based on experimental studies, we made the following conclusions.

1. A two-component film, containing methane clathrates, forms in the process of cryovacuum co-condensation of water vapor and methane on the substrate at $T=16 \mathrm{~K}$.

2. The behavior of the samples during the temperature increase depends on water and methane concentration ratios. However, at the characteristic temperature ranges, which are associated with the equilibrium properties of methane and phase structural transformations of amorphous solid water, main features of the character of measured parameters change for all samples are the same.

3. The samples contain methane until ice evaporation at the temperature of about $T=180 \mathrm{~K}$. This may serve as confirmation of the assumption of methane clathrates presence in the studied samples.

1. A. Drobyshev, A. Aldiyarov, K. Katpaeva, E. Korshikov, V. Kurnosov, and D. Sokolov, Fiz. Nizk. Temp. 39, 919 (2013) [Low Temp. Phys. 39, 714 (2013)].

2. A. Aldiyarov, A. Drobyshev, E. Korshikov, V. Kurnosov, and D. Sokolov, Phys. Solid State 54, 1475 (2012). 
3. P. Jenniskens and D.F. Blake, Astrophys. J. 473, 1104 (1996).

4. A. Aldiyarov, M. Aryutkina, A. Drobyshev, M. Kaikanov, and V. Kurnosov, Fiz. Nizk. Temp. 35, 333 (2009) [Low Temp. Phys. 35, 251 (2009)].

5. C. Talon, M. Ramos, S. Vieira, G. Guello, F. Bermejo, A. Griado, M. Senent, S. Bennington, H. Fischer, and H. Schober, Phys. Rev. 58, 745 (1998).

6. M.E. Fajardo and S. Tam., J. Chem. Phys. 115, 6807 (2001).

7. A.J. Tursi and E.R. Nixon, J. Chem. Phys. 52, 1521 (1970).

8. J.B. Paul, C.P. Collier, R.J. Saykally, J.J. Sherer, and A.O. Keefe, J. Phys. Chem. 101, 5211 (1997).

9. V. Manzhelii and Y. Freiman. Physics of Cryocrystals, AIP, Woodbury, New York (1996).
10. A. Drobyshev, A. Aldiyarov, D. Zhumagaliuly, V. Kurnosov, N. Tokmoldin. Fiz. Nizk. Temp. 33, 627 (2007) [Low Temp. Phys. 33, 472 (2007)].

11. P.G. Debenedetti, J. Phys. Condence Matter 15, 1670 (2003).

12. G.P. Johari, A. Hallbrucker, and E. Mayer, Science 273, 90 (1996).

13. A. Drobyshev, A. Aldiyarov, D. Zhumagaliuly, V. Kurnosov, and N. Tokmoldin, Fiz. Nizk. Temp. 33, 479 (2007) [Low Temp. Phys. 33, 355 (2007)].

14. G.P. Johari, J. Chem. Phys. 119, 2935 (2003). 